\title{
ON ALGEBRAIC CLOSURE IN FUNCTION ALGEBRAS
}

\author{
DON DECKARD AND CARL PEARCY
}

1. Introduction. We say that the algebra $C(\mathscr{X})$ of all continuous complex-valued functions on a topological space $x$ is algebraically closed if every monic polynomial equation with coefficients from $C(x)$ has a solution in $C(x)$. In [1] the authors proved that if $x$ is a Stonian space (i.e., a compact Hausdorff space such that the closure of every open set in $x$ is open), then $C(x)$ is algebraically closed. In this note we generalize this result to the case in which $x$ is assumed to be only a totally disconnected, compact Hausdorff space, and then we prove the same theorem for the case in which $x$ is a compact interval on the real line. Finally, we indicate how the same techniques can be applied to show that if $X$ is any (not necessarily compact) linearly ordered, order-complete space, then $C(x)$ is algebraically closed.

2. We begin with a useful lemma.

Lemma 2.1. If $x$ is a totally disconnected, compact Hausdorff space, and $\mathcal{U}$ is an open subset of $X$ which is also an $F_{\sigma}$, then $\mathcal{U}$ is the disjoint union of a countable family of compact open subsets of $x$.

Proof. Write $\mathfrak{U}=\cup_{n=1}^{\infty} \mathfrak{e}_{n}$, where the $\mathfrak{e}_{n}$ are closed in $x$. Using the Heine-Borel property and the fact that $x$ is totally disconnected, one can obtain a compact open set $D_{1}$ such that $u \supset D_{1} \supset \mathfrak{C}_{1}$. Then $D_{1}^{\prime} \cap \mathfrak{C}_{2}$ is closed, and one can obtain a compact open set $D_{2}$ satisfying $\mathcal{U} \supset D_{2} \supset D_{1}^{\prime} \cap \mathfrak{C}_{2}$ and $\mathscr{D}_{2} \cap D_{1}=\varnothing$. The argument proceeds by induction in the obvious fashion.

Because of the frequency with which we use it in what follows, we state without proof Lemma 2.2 of [1].

Lemma 2.2. Suppose $\mathfrak{J}$ is any topological space, and $P(x, z)=z^{n}$ $+a_{n-1}(x) z^{n-1}+\cdots+a_{0}(x)$, where the $a_{i} \in C(\mathfrak{J})$. If $z_{0}$ is a root of multiplicity $\mu$ of the polynomial equation $P\left(x_{0}, z\right)=0$, and if $\epsilon>0$ is such that $P\left(x_{0}, z\right)$ has no zero other than $z_{0}$ satisfying $\left|z-z_{0}\right|<\epsilon$, then there is a neighborhood $\mathfrak{N}\left(x_{0}, \epsilon\right)$ of $x_{0}$ such that for $x \in \mathfrak{N}\left(x_{0}, \epsilon\right), P(x, z)$ $=0$ has exactly $\mu$ roots (counting multiplicities) satisfying $\left|z-z_{0}\right|<\epsilon$.

We next prove

Theorem 1. Suppose that $x$ is a totally disconnected, compact Hausdorff space, and let $P(x, z)=z^{n}+a_{n-1}(x) z^{n-1}+\cdots+a_{0}(x)$, where the $a_{i} \in C(x)$. Then there is an $f \in C(x)$ such that $P(x, f(x)) \equiv 0$.

Received by the editors November 27, 1962. 
Proof. Since $X$ is compact and has a base of compact open sets, it is clear that it suffices to prove the following local result: $\left(^{*}\right)$ if $x_{0} \in \mathcal{X}$ and $P\left(x_{0}, z_{0}\right)=0$, then there exist a neighborhood $\mathfrak{I}\left(x_{0}\right)$ and an $f \in C\left(\mathscr{T}\left(x_{0}\right)\right)$ such that $f\left(x_{0}\right)=z_{0}$ and $P(x, f(x)) \equiv 0$ on $\mathscr{N}\left(x_{0}\right)$. We obtain this result by inducing on the multiplicity $\mu\left(z_{0}\right)$ of the root $z_{0}$ of $P\left(x_{0}, z\right)=0$. More explicitly, the induction hypothesis is the following: if $\tau>0, x_{0} \in \mathcal{X}, P\left(x_{0}, z_{0}\right)=0$, and $\mu\left(z_{0}\right)=k$, then there exist a neighborhood $\mathscr{N}\left(x_{0}\right)$ and an $f \in C\left(\mathscr{N}\left(x_{0}\right)\right)$ such that $f\left(x_{0}\right)=z_{0}$ and such that for $x \in \Re\left(x_{0}\right), P(x, f(x))=0$ and $\left|f(x)-z_{0}\right|<\tau$. If $\mu\left(z_{0}\right)=1$, Lemma 2.2 tells us that if $\epsilon>0$ is chosen such that $P\left(x_{0}, z\right)=0$ has no root other than $z_{0}$ satisfying $\left|z-z_{0}\right|<\epsilon$, then there is a neighborhood $\mathfrak{N}\left(x_{0}\right)$ such that for $x \in \mathscr{N}\left(x_{0}\right), P(x, z)$ has exactly one root-say $f(x)$-satisfying $\left|f(x)-z_{0}\right|<\epsilon$. The fact that the function $f$ so defined on $\mathfrak{I}\left(x_{0}\right)$ is continuous on $\Re\left(x_{0}\right)$ is proved as follows. Given $x_{1} \in \mathfrak{N}\left(x_{0}\right)$ and $\rho<\epsilon-\left|f\left(x_{1}\right)-z_{0}\right|$, apply Lemma 2.2 to obtain a neighborhood $\Re\left(x_{1}\right) \subset \Re\left(x_{0}\right)$ such that for $x \in \Re\left(x_{1}\right), P(x, z)=0$ has exactly one root $r(x)$ satisfying $\left|r(x)-f\left(x_{1}\right)\right|<\rho$. Since $f(x)$ is the only zero of $P(x, z)$ satisfying $\left|f(x)-z_{0}\right|<\epsilon, \quad r(x)=f(x)$, or in other words, $\left|f(x)-f\left(x_{1}\right)\right|<\rho$. Thus $f$ is continuous on $\mathscr{N}\left(x_{0}\right)$. Now suppose that we have verified the induction hypothesis for all points $x \in X$ and zeros $z$ of $P(x, z)$ such that $\mu(z)<k$, and let $x_{0} \in X$ be such that $P\left(x_{0}, z_{0}\right)=0$ and $\mu\left(z_{0}\right)=k$.

We again apply Lemma 2.2 with $\epsilon$ as above to obtain a compact open neighborhood $\Re\left(x_{0}\right)$ such that for $x \in \Re\left(x_{0}\right), P(x, z)=0$ has exactly $k$ roots (counting multiplicities) satisfying $\left|z-z_{0}\right|<\epsilon$. Furthermore, we can arrange it so that for $x \in \mathfrak{N}\left(x_{0}\right), P(x, z)$ has no zero satisfying $\left|z-z_{0}\right|=\epsilon$. Let $P_{m} \subset \Re\left(x_{0}\right)$ be the set of points $x$ for which $P(x, z)=0$ has at least two distinct roots which are at a distance $\geqq 1 / m$ from each other and which lie in the disc $\left\{z:\left|z-z_{0}\right|<\epsilon\right\}$. Let $\mathbb{P}=\bigcup_{m=1}^{\infty} P_{m}$. (Thus for $x \in \Re\left(x_{0}\right)-\mathcal{P}, P(x, z)=0$ has exactly one root of multiplicity $k$ satisfying $\left|z-z_{0}\right|<\epsilon$.) An easy application of Lemma 2.2 shows that $P$ is open. We show that each $P_{m}$ is closed (some may be void), and thus that $\odot$ is an $F_{\sigma}{ }^{1}$

If $P_{m}$ is nonvoid, let $x_{\lambda}$ be a net in $P_{m}$ such that $x_{\lambda} \rightarrow x_{1}$, and for each $\lambda$, let $z_{1}(\lambda)$ and $z_{2}(\lambda)$ be zeros of $P\left(x_{\lambda}, z\right)$ satisfying $\left|z_{1}(\lambda)-z_{2}(\lambda)\right|$ $\geqq 1 / m$ and $\left|z_{i}(\lambda)-z_{0}\right|<\epsilon$. Clearly, one can choose a subnet $x_{\beta}$ of $x_{\lambda}$ with the property that the nets $z_{1}(\beta)$ and $z_{2}(\beta)$ converge-say to $z_{1}$ and $z_{2}$, respectively. Then, since $P(x, z)$ is jointly continuous in $x$ and $z, P\left(x_{1}, z_{1}\right)=P\left(x_{1}, z_{2}\right)=0$, and $\left|z_{1}-z_{2}\right| \geqq 1 / m$. Also, since by previous arrangement we cannot have $\left|z_{i}-z_{0}\right|=\epsilon$, we must have $\left|z_{i}-z_{0}\right|<\epsilon$ for $i=1,2$. Thus $x_{1} \in \mathcal{P}_{m}$, so $\mathcal{P}_{m}$ is closed. We wish to con-

1 This observation is due to Arlen Brown, who conjectured that our result in [1] could be generalized. 
struct an $f \in C\left(\Re\left(x_{0}\right)\right)$ such that $f\left(x_{0}\right)=z_{0}$ and such that for $x \in \mathscr{N}\left(x_{0}\right)$, $P(x, f(x))=0$. (The other requirement of the induction hypothesis to be met, namely $\left|f(x)-z_{0}\right|<\tau$ for $x \in \Re\left(x_{0}\right)$, can then be satisfied by dropping down to a smaller neighborhood of $x_{0}$ if necessary.) We first construct an appropriate $f$ on $\mathcal{P}$, and then define $f$ on $\Re\left(x_{0}\right)-\mathcal{P}$. It follows from Lemma 2.1 that $\beta$ can be written $\rho=\bigcup_{m=1}^{\infty} \mathfrak{C}_{m}$, where the $\mathcal{C}_{m}$ are mutually disjoint compact open sets. Let $m$ be arbitrary but fixed. Then for $x \in \mathrm{C}_{m}$, there is a zero $z(x)$ of $P(x, z)$ satisfying $\mu(z(x))<k$ and $\left|z(x)-z_{0}\right|<\epsilon$. Thus using the induction hypothesis (with $\tau=\epsilon-\left|z(x)-z_{0}\right|$ ) and the fact that $\mathfrak{C}_{m}$ is compact open, we construct a function $f_{m} \in C\left(\mathcal{e}_{m}\right)$ such that for $x \in \mathcal{C}_{m}, P\left(x, f_{m}(x)\right)=0$ and $\left|f_{m}(x)-z_{0}\right|<\epsilon$. Hence the function $f$ defined on $P$ so as to extend all of the $f_{m}$ is such that $f \in C(P)$ and such that for $x \in P, P(x, f(x))=0$ and $\left|f(x)-z_{0}\right|<\epsilon$. To complete the proof of the theorem, we wish to define $f$ on $\mathscr{N}\left(x_{0}\right)-P$ so that the extension is in $C\left(\mathscr{N}\left(x_{0}\right)\right)$. For $x \in \Re\left(x_{0}\right)$ $-P$, there is exactly one root (of multiplicity $k$ ) of $P(x, z)=0$ satisfying $\left|z-z_{0}\right|<\epsilon$. Define $f(x)$ to be this root. The fact that $f$ is continuous at $x \in \Re\left(x_{0}\right)-\odot$ follows from another application of Lemma 2.2, and we leave this verification to the reader.

3. In this section we consider the case in which $X$ is a compact interval-say $[0,1]$-on the real line. The proof that $C([0,1])$ is algebraically closed uses the following lemma.

Lemma 3.1. Suppose $P(x, z)$ is as before with the $a_{i} \in C([0,1])$, and suppose $r \in(0,1]$. If $f \in C([0, r))$ is such that $P(x, f(x))=0$ for $x \in[0, r)$, then $\lim _{x \rightarrow r-} f(x)$ exists and is a root of $P(r, z)=0$.

Proof. Let $z_{1}, \cdots, z_{k}$ be the distinct roots of $P(r, z)=0$, and choose $\epsilon>0$ so that the $\operatorname{discs} D_{i}=\left\{z:\left|z-z_{i}\right|<\epsilon\right\}$ are mutually disjoint. By applying Lemma 2.2, we obtain a neighborhood $\mathfrak{N}(r)$ of $r$ such that for $x \in \Re(r)$, every zero of $P(x, z)$ is in some $\operatorname{disc} D_{i}$. Choose $s \in \mathfrak{N}(r)$ such that $s<r$. Then $f(s)$ must lie in one of the discs $D_{i}-$ say $f(s) \in D_{i_{0}}$. It follows that for $s<x<r, f(x)$ must lie in the same disc $D_{i_{0}}$, and repeating the above argument with any $\delta<\epsilon$ in the place of $\epsilon$ shows that $\lim _{x \rightarrow r-} f(x)=z_{i_{0}}$.

TheOREM 2. The algebra $C([0,1])$ is algebraically closed.

Proof. Let $P(x, z)$ be as above. Taking into account Lemma 3.1 and the compactness of $[0,1]$, it clearly suffices to prove the local result $\left({ }^{*}\right)$ which appeared in the proof of Theorem 1 . To establish $\left(^{*}\right)$, we again induce on the multiplicity $\mu\left(z_{0}\right)$ of the zero $z_{0}$ of $P\left(x_{0}, z\right)$, and the induction hypothesis is the same as before. The proof now proceeds essentially just as in Theorem 1 until the open set $\odot \subset \Re\left(x_{0}\right)$ is obtained. (The neighborhood $\Re\left(x_{0}\right)$ is chosen to be an open interval unless $x$ is 0 or 1 , in which case $\mathfrak{N}\left(x_{0}\right)$ is chosen to be a half open 
interval.) If an $f \in C(\mathcal{P})$ can be constructed such that for $x \in \mathcal{P}$, $P(x, f(x))=0$ and $\left|f(x)-z_{0}\right|<\epsilon$, then the proof is completed by defining $f$ on $\Re\left(x_{0}\right)-\odot$ just as in the proof of Theorem 1. To construct such an $f \in C(P)$, it suffices to construct $f$ (with the appropriate properties) on the disjoint open intervals whose union is $P$. Let $(s, t)$ be such an open interval, and consider the collection $F$ of ordered pairs $\left\langle\left(x_{1}, x_{2}\right), g\right\rangle$, where $\left(x_{1}, x_{2}\right) \subset(s, t)$ and $g \in C\left(\left(x_{1}, x_{2}\right)\right)$ is such that for $x \in\left(x_{1}, x_{2}\right), P(x, g(x))=0$ and $\left|g(x)-z_{0}\right|<\epsilon$. Since $(s, t) \subset \mathfrak{N}\left(x_{0}\right)-\odot$, the induction hypothesis can be applied at any point of $(s, t)$ to show that $F$ is nonvoid. Define a partial ordering on $F$ by setting $\left\langle\left(x_{1}, x_{2}\right), g\right\rangle$ $\leq\left\langle\left(x_{3}, x_{4}\right), h\right\rangle$ if and only if $\left(x_{1}, x_{2}\right) \subset\left(x_{3}, x_{4}\right)$ and $h$ is an extension of $g$. Clearly, chains in $F$ have upper bounds, so by Zorn's Lemma, $F$ contains a maximal element $\left\langle\left(y_{1}, y_{2}\right), f\right\rangle$.

The proof is completed by showing that $\left(y_{1}, y_{2}\right)=(s, t)$. If $\left(y_{1}, y_{2}\right)$ $\neq(s, t)$, the induction hypothesis, together with Lemma 3.1, can be applied to the appropriate point $y_{1}$ or $y_{2}$ to obtain a contradiction to the maximality of $\left\langle\left(y_{1}, y_{2}\right), f\right\rangle$.

Corollary. If $x$ is the entire real line, then $C(x)$ is algebraically closed.

Proof. By the above theorem, construct $n$ root functions on each interval $[n, n+1]$, write $X=\cup_{n=-\infty}^{\infty}[n, n+1]$, and patch.

The connectedness of the real line was used in Lemma 3.1, but the fact is that by doing things slightly differently and going to somewhat more trouble, the use of connectedness can be avoided, and the following theorem can be proved.

THEOREM 3. If $X$ is any linearly ordered, order-complete topological space, then $C(X)$ is algebraically closed. (A space is order-complete if every nonvoid subset which is bounded above has a supremum.)

SKETCH OF THE PROOF. There is no loss of generality in assuming that $X$ has a first point and has no last point. The following known fact makes it sufficient to prove that if $x_{0}, y_{0} \in \mathcal{X}$ with $x_{0}<y_{0}$, then there exist $f_{1}, \cdots, f_{n} \in C\left(\left[x_{0}, y_{0}\right]\right)$ such that on $\left[x_{0}, y_{0}\right], P(x, z)$ $\equiv\left(z-f_{1}(x)\right)\left(z-f_{2}(x)\right) \cdots\left(z-f_{n}(x)\right)$. Fact: If $x$ is a linearly ordered, order-complete space possessing no last point, then either (a) $x$ contains a cofinal $\omega$-sequence or (b) given any $f \in C(x)$, there is an $x_{f} \in \mathscr{X}$ to the right of which $f$ is constant. To obtain the above-mentioned result concerning intervals $\left[x_{0}, y_{0}\right]$, one first proves a local result like $\left.{ }^{*}\right)$ in the proof of Theorem 1, except that the existence of $n$ "desirable" functions on $\mathfrak{N}\left(x_{0}\right)$ is asserted. One proves this local result by induction as above. The induction hypothesis explicitly is as follows: If $x_{0} \in X, P\left(x_{0}, z_{0}\right)=0, \mu\left(z_{0}\right)=k$, and $\epsilon>0$ is such that the only zero 
of $P\left(x_{0}, z\right)$ satisfying $\left|z-z_{0}\right|<\epsilon$ is $z_{0}$ itself, then there exist $\mathfrak{N}\left(x_{0}\right)$ and $f_{1}, \cdots, f_{k} \in C\left(\mathfrak{N}\left(x_{0}\right)\right)$ such that for $x \in \mathfrak{N}\left(x_{0}\right)$, the numbers $f_{1}(x), \cdots$, $f_{k}(x)$ are exactly the roots of $P(x, z)=0$ in the open $\epsilon$-disc about $z_{0}$. The verification of the induction hypothesis for $\mu\left(z_{0}\right)=k$ goes along essentially as in Theorem 1 until the open set $P \subset \Re\left(x_{0}\right)$ is obtained. $\left(\mathscr{N}\left(x_{0}\right)\right.$ is taken to be open.) Just as before, it suffices to construct the $k$ functions having the desired properties on $\beta$, or even on any of the disjoint open intervals whose union is $P$. But such an open interval $\left(x_{1}, x_{2}\right)$ is itself a linearly ordered, order-complete space, so the same considerations mentioned at the beginning of this outline apply, and it suffices to be able to construct the $k$ functions on every closed interval $\left[x_{3}, x_{4}\right] \subset\left(x_{1}, x_{2}\right)$. To construct the $k$ functions on $\left[x_{3}, x_{4}\right]$, the induction hypothesis is applied at $x_{3}$ to get one started. Then one considers intervals $\left[x_{3}, y\right)$ on which one can do business and proves by a patching argument (involving $k$ functions) that the supremum of such $y$ lies to the right of $x_{4}$. Throughout the argument, care must be exercised because a point can be isolated from the left or right.

\section{Remarks.}

(1) Theorem 2, which is very useful in certain eigenvalue problems of mathematical physics, is not new. However, the authors have been unable to locate a proof of the theorem in the literature.

(2) Obviously, there are linearly ordered, order-complete spaces which are not totally disconnected. G. S. Young [2] has recently pointed out that there are compact Hausdorff, totally disconnected spaces which cannot be linearly ordered. Thus neither Theorem 1 nor Theorem 3 implies the other.

(3) One would like to classify (topologically) the compact Hausdorff spaces $x$ with the property that $C(x)$ is algebraically closed. In this connection it is easy to see that if $X$ contains the homeomorphic image of the unit circle, then $C(x)$ cannot be algebraically closed. Also, there are compact subsets $X$ of the plane which contain no homeomorphic image of the circle and for which $C(x)$ is not algebraically closed. (For example, let $x$ consist of the graph of the function $y=\sin (1 / x), 0<x \leqq 2 \pi$, together with the segment $\{(0, y):-1 \leqq y \leqq 1\}$ of the $y$-axis. $)$

\section{REFERENCES}

1. D. Deckard and C. Pearcy, On matrices over the ring of continuous complexvalued functions on a Stonian space, Proc. Amer. Math. Soc. 14 (1963), 322-328.

2. G. S. Young, Representations of Banach spaces, Proc. Amer. Math. Soc. 13 (1962), 667-668.

RICE UNIVERSITY AND

Humble Oil and Refining Company 